\title{
AN ELEMENTARY PROOF OF THE CLASSIFICATION OF SURFACES IN THE PROJECTIVE 3-SPACE
}

\author{
J. H. C. CREIGHTON
}

\begin{abstract}
A closed surface embeds in the 3-dimensional real projective space if and only if it is orientable or of odd Euler characteristic. The proof given is elementary in the sense that only geometric techniques are used.
\end{abstract}

The seemingly elementary fact that the Klein bottle does not embed in $R P^{3}$, the 3-dimensional real projective space, seems to have been first known to Stiefel [3]. Later Steenrod gave a proof (unpublished) using a delicate and rather lengthy fundamental group argument. In general, the classification of surfaces in $R P^{3}$ is given by the following theorem.

THEOREM 1. $A$ closed surface $M$ embeds in $R P^{3}$ if and only if $M$ is orientable or of odd Euler characteristic.

This theorem was given explicitly in 1969 by Bredon and Wood [1] as a corollary of the classification of nonorientable surfaces in 3-dimensional lens spaces. Note that the result for the Klein bottle is not sufficient to prove the theorem, for one cannot 'remove handles' from an embedding. For example, (see [1]) the Klein bottle does not embed in $N \times S^{1}, N$ an orientable surface, but with one handle it does embed. Theorem 1 is also obtained in [2] as a corollary of the cobordism classification of hypersurfaces in higher dimensional lens spaces. However, both of these latter proofs rely heavily on cobordism theory with the associated machinery of algebraic topology and homotopy theory.

In this note we provide an elementary proof of Theorem 1. The proof is elementary in the sense that we use only geometric techniques, a series of surgeries which preserve the parity of the Euler characteristic. Nevertheless, the theorem is not to be considered elementary. Our proof is merely an elementary reduction to the nonelementary fact that a surface in the 3-sphere is orientable.

Let $R P^{3}$ with the standard $R P^{2}$ be determined by the following diagram

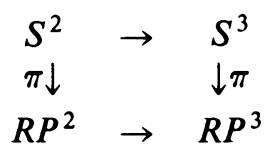

Received by the editors June 14, 1977.

AMS (MOS) subject classifications (1970). Primary 57D40.

Key words and phrases. Real projective 3-space, surgery. 
and assume $M$ transverse to $R P^{2}$. Let $\bar{M}=\pi^{-1} M$.

LEMMA. If $M$ is nonorientable and $M \cap R P^{2}$ is connected, then $\bar{M} \cap S^{2}$ is connected.

Proof. We show that $S^{2}-\bar{M}$ has two (not three) components. Let $S^{3} \stackrel{A}{\rightarrow} S^{3}$ be the antipodal map. Then $\left.A\right|_{\bar{M}}$ is orientation reversing. But on $S^{3}, A$ is orientation preserving. Thus if $\bar{M}_{0}$ and $\bar{M}_{1}$ are the components of $S^{3}-\bar{M}$ then $A \bar{M}_{i}=\bar{M}_{1-i}$. That is, $\bar{M}_{0}$ and $\bar{M}_{1}$ are diffeomorphic under $A$. Thus $S^{2} \cap \bar{M}_{0}$ and $S^{2} \cap \bar{M}_{1}$ are diffeomorphic under $A$. Thus the components of these two sets, which are just the components of $S^{2}-\bar{M}$, are even in number.

Proof OF THE THEOREM. It suffices to show that for a nonorientable surface $M$ embedded in $R P^{3}$ transverse to $R P^{2}$, the Euler characteristic is odd. By attaching handles, we may assume $M \cap R P^{2}$ connected. At this point it is convenient to regard $R P^{3}$ as obtained from the 3-ball as in the diagram

$$
\begin{array}{ccc}
S^{2} & \rightarrow & B^{3} \\
\pi \downarrow & & \downarrow \pi \\
R P^{2} & \rightarrow & R P^{3}
\end{array}
$$

where $\pi$ is a diffeomorphism off of $S^{2}$. In $B^{3}$ choose a tubular neighborhood of $S^{2}, \bar{T}=S^{2} \times[0,1]$ for which $\bar{M} \cap\left(S^{2} \times t\right)$ are diffeomorphic for all $t$. Then $T=\pi \bar{T}$ is a tubular neighborhood of $R P^{2}$. Note that $\bar{M} \cap \bar{T}$ is a cylinder (since $\bar{M} \cap S^{2}$ is connected) and so $M \cap T$ is a Möbius band. Let $\Sigma$ be the boundary sphere of $T$. Cut $\Sigma$ and $M$ along $M \cap \Sigma$ and paste as follows:

(a) match one disk of $\Sigma-M$ to $M \cap T$, to obtain a projective plane,

(b) match the other disk to $M-T$, to obtain an orientable surface; orientable because embedded away from $R P^{2}$.

We have now performed the following operations on $M$, preserving the parity of the Euler characteristic:

$$
\begin{aligned}
M & \rightarrow M \text { with handles }=M_{w h}, \\
& \rightarrow M_{w h}+\Sigma, \\
& \rightarrow \text { projective plane }+0,
\end{aligned}
$$

where 0 is an orientable surface. Thus the Euler characteristic of $M$ is odd.

\section{BIBLIOGRAPHY}

1. G. E. Bredon and J. W. Wood, Non-orientable surfaces in orientable 3-manifolds, Invent. Math. 7 (1969), 83-110.

2. J. H. C. Creighton, Hypersurfaces in lens spaces (to appear).

3. E. Stiefel, Richtungsfelder und Fernparallelismus in n-dimensionalen Mannigfaltigkeiten (Anhang I, §2), Comment. Math. Helv. 8 (1935), 305-353.

Department of Mathematics, Pahlavi University, Shiraz, Iran

Current address: c/o Weedon, 1517 Auburn Avenue, Rockville, Maryland 20850 